\title{
Artigo
}

\section{Estudos em Políticas Públicas e a COVID-19: indicativos de uma agenda de pesquisa}

Felipe Gonçalves Brasil ${ }^{1}$

\section{Resumo}

A pandemia causada pela Covid-19, que se prolonga desde os primeiros meses de 2020 ao redor de todo o mundo, marcada por irreparáveis impactos sociais, econômicos, sanitários e humanitários, tem despertado a atenção de acadêmicos que se dedicam a entender e analisar as ideias, escolhas e prioridades dos tomadores de decisão num momento caracterizado pela extrema necessidade de atuação governamental. Seja no seu papel de fonte oficial de informações que orientem e informem a população, seja na atuação direta na elaboração de estratégias de redução do contágio, no estabelecimento de regras de funcionamento de equipamentos públicos e privados, ou na elaboração de políticas que minimizem os efeitos catastróficos da pandemia e garantam a sobrevivência de seu povo, o "estado em ação" vem sendo observado por diferentes lentes teóricas inseridas no interdisciplinar campo das políticas públicas. Este estudo tem o objetivo de apresentar, ainda que brevemente, dois referenciais teóricos e analíticos com grande potencial para apoiar estudos que buscam entender melhor a forma como o processo de políticas públicas pode sofrer importantes alterações em momentos de crise como essa. 0 primeiro referencial analisado é o dos "efeitos focalizadores" (focusing events), presentes na literatura de policy process e agenda-setting. Outra lente analítica relevante está relacionada com o aprendizado em políticas públicas e a formulação de políticas baseadas em evidência ( policy learning and evidencebased policy making). As escolhas por essas três linhas teóricas não têm a intenção de limitar os estudos em políticas públicas, mas de lançar luz a novas abordagens com grande potencial explicativo para novas agendas de pesquisas.

\section{Palavras-chave}

Cidades. Espaços. Poder. Conflitos. Simbolismos.

\footnotetext{
${ }^{1}$ Felipe Gonçalves Brasil é pós-doutorando e docente no Departamento de Administração Pública da UNESP Araraquara. E-mail: fbrasil.pp@gmail.com.
} 


\section{Abstract}

The pandemic caused by COVID-19, which has been going on since the first months of 2020 around the world, marked by irreparable social, economic, health and humanitarian impacts, has attracted the attention of academics dedicated to understanding and analyzing ideas, choices and priorities of decision makers at a time characterized by the extreme need for governmental action. Be it in its role as an official source of information to guide and inform the population, be it in the direct action of developing strategies to reduce infection, establishing operating rules for the public and private facilities, or in the policy-making that minimizes the catastrophic effects caused by the pandemic and guarantees the survival of its people, the "state in action" has been observed through different theoretical lenses inserted in the interdisciplinary field of public policies. This study aims to present, albeit briefly, two theoretical and analytical frameworks with great potential to support studies that seek to better understand how the public policy process can undergo important changes in times of crisis. The first framework analyzed is focusing events, which is present in the studies of John Kingdon (1984) and Thomas Birkland (2003). Another relevant analytical lens is related to policy learning and evidence-based policy making. The choices for these three theoretical lines are not intended to limit studies in public policies, but to shed light on new approaches with great explanatory potential for new research agendas.

\section{Keywords}

Focusing events. Public policy learning. Evidence-based policy making. COVID-19 pandemic. Public policy analysis.

\section{Introdução}

Os efeitos da pandemia causada pela Covid-19 são múltiplos e, em muitos casos, irreversíveis. Até dezembro de 2020, somam-se mais de 1,5 milhão de mortes em todo o mundo. Diversos são os exemplos de consequências gravíssimas que afetaram o dia a dia das pessoas e de mudanças às quais as sociedades tiveram que se adaptar para sobreviver em tempos de pandemia. Dos prejuízos econômicos causados pelo lockdown e pelas inúmeras (e necessárias) políticas de restrição às atividades econômicas, do varejo e no mundo do trabalho, aos impactos sociais causados pelo distanciamento social, pelas mudanças de rotina, fechamento de escolas, home-office, acesso à internet e equipamentos tecnológicos, perpassando por temas humanitários, como o aumento da violência doméstica, a possibilidade de sobrevivência e a própria saúde mental, a pandemia tem nos mostrado que crises como essa mudam a 
forma, a velocidade a até a ordem como as questões são entendidas e priorizadas no competitivo processo de políticas públicas.

A emergência pela tomada de decisões e a dependência de ações governamentais que tenham potencial de atingir grandes parcelas da população num curto espaço de tempo são desafios que modificam e redefinem a lógica e as formas como os policymakers atuam no processo de produção de políticas. O tempo, um recurso escasso e de grande importância, é uma variável de primeira ordem a ser considerada em momentos de crises. A urgência altera a forma como as informações são processadas e reduz as fontes confiáveis que geram, coordenam e difundem tais informações. Os atores políticos são pressionados a se posicionarem e atuarem de forma imediata, rompendo com o modus operandi de formação de coalizões e do jogo de barganha, comuns em processos decisórios e pré-decisórios, de estabelecimento de prioridades e seleção de alternativas.

Nesse período de crise marcado pela necessidade de ação, informação e atenção, a mídia figura entre um dos atores mais importantes quando consideramos a capacidade de produção e difusão de informação, afetando, também, a atenção da opinião pública e até a forma como os atores governamentais entendem e priorizam os problemas e as soluções decorrentes do evento em questão. Estudos que destacam a relação das agendas (midiática, da opinião pública e governamental) em momentos de crise não são novidade no campo das políticas públicas. Na verdade, eles são parte do surgimento dos estudos de agenda-setting, com a proposição de estudos que analisam os efeitos da mídia na opinião pública no pós-guerra. Traquina (1995) retoma o surgimento de novos meios de comunicação do período vinculando-os a efeitos que as notícias veiculadas (agenda midiática) poderiam causar no público (agenda da opinião pública). Já em 1922, Lippmann demonstrava a preocupação pelo fato de a mídia ter o poder de apresentar imagens para o público. Ainda que não utilizasse a expressão agenda-setting, foi quem primeiro descreveu as relações existentes entre mídia e agenda pública, hoje entendida como o processo de agendamento da agenda pública. A mass media surgia, portanto, como um objeto de estudo importante que apontava a relação entre a seleção daquilo que era noticiado e a forma como o público se importava e recebia tais informações. Entre as décadas de 1940 a 1970, nos Estados Unidos, sobretudo, se intensificaram os estudos dos mass media que buscavam relacionar causa e efeito entre as múltiplas agendas com foco no âmbito eleitoral (LAZARFELD; BERELSON; GAUDET, 1944, 1955; KLAPPER, 1960). Os trabalhos de Cohen (1963), sobretudo com a pesquisa empírica de McComb 
e Shaw (1972), no início dos anos 1970, fizeram com que o conceito de agendasetting ganhasse destaque e passasse a ser empiricamente investigado nos Estados Unidos.

É com John Kingdon (1984), na década seguinte, que os estudos sobre formação de agenda ganham novos contornos e destaque no cenário americano e internacional. Os estudos de agenda-setting buscam entender de que maneira alguns temas são priorizados pelos tomadores de decisão enquanto diversas outras questões são deixadas em segundo plano ou mesmo sequer são percebidas como sendo problemas públicos. A formação de agenda é, portanto, um processo competitivo no qual diversos temas disputam a atenção e um lugar na restrita e limitada agenda dos tomadores de decisão. Baseado em estudos anteriores de dentro da policy science, propostos por Cobb e Elder (1972), três contribuições são relevantes para a compreensão do processo de formação da agenda: 1- problemas públicos são construções sociais e não fatos dados, imutáveis e inquestionáveis. A compreensão de um problema depende, sobretudo, da realidade percebida e moldada sobre essa questão, podendo transformá-la, ou não, em um problema público que demande a ação do Estado; 2 - o processo de definição de problemas é marcado pelo duelo de distintos grupos de pressão, que se utilizam de valores e símbolos para formular, contestar e redefinir a imagem de problemas públicos. Com o objetivo de ganhar vantagens no processo de formulação de políticas, a forma como cada problema é definido, bem como a forma como tantas outras questões são descreditadas e ocultadas no processo pré-decisório, são estratégias de atuação dos atores envolvidos no processo de expansão do conflito e na criação de problemas públicos. Essa luta pela definição do problema não apenas serve como forma estratégica de barganha e privilégios no acesso aos tomadores de decisão, mas também como um mecanismo de defesa dos grupos organizados contra a entrada de novos participantes no jogo político. $\mathrm{O}$ acesso às arenas pré-decisórias por novos atores pode apresentar eventos inesperados e mudanças de valores dentro das comunidades de políticas (CAPELLA; BRASIL, 2016); 3 - Cobb e Elder (1972) são os primeiros autores da literatura a sistematizar o processo de agendamento por meio da criação de duas etapas de agendas: a agenda sistêmica e a agenda institucional, especificando um processo fluido e em constante mudança no trânsito de temas. Os autores buscam entender, portanto, de que forma os problemas são construídos e como eles se tornam prioritários na agenda governamental. De acordo com Capella (2007, p. 45): 
Os estudos sobre a agenda governamental mostram que a complexidade do processo decisório e o volume de questões que se apresentam sistematicamente aos formuladores de políticas acabam por concentrar a atenção destes em um conjunto limitado de assuntos considerados relevantes. Alguns dos assuntos que sobrevivem a esse competitivo processo de seleção podem vir a integrar uma política pública, ou seja, podem ser transformados em programas e projetos governamentais. Uma das preocupações centrais ao estudo de agenda-setting é, portanto, compreender como as questões capturam a atenção dos formuladores de políticas.

John Kingdon (1984) foi o primeiro autor a propor um modelo analítico sintético para estudar o processo de priorização de assuntos e de formação da agenda governamental. A obra de Kingdon apresenta contribuições importantes para a construção do campo de análise para além do seu conhecido modelo de Múltiplos Fluxos. O autor também destaca o papel dos empreendedores de políticas públicas, a importância das comunidades de políticas públicas, além de apontar para uma série de mecanismos pelos quais, segundo o autor, funcionaria o processo como um problema é percebido até a efetiva formulação de uma política pública. A primeira lente analítica proposta por esse trabalho como um indicativo de agenda para estudos sobre a pandemia destaca o papel dos eventos focalizadores no processo de reconhecimento de problemas e formação da agenda governamental. Prevista na elaboração inicial de Kingdon (1984) e ampliada em estudos posteriores por Birkland (1997, 2005, 2006), os eventos focalizadores são entendidos como de grande impacto e magnitude, que alteram significativamente a forma pela qual os atores disputam o reconhecimento e a construção da imagem de problemas públicos.

\section{Eventos Focalizadores}

A literatura de políticas públicas já reservava, desde o início de anos 1980, com a obra de Kingdon (1984) atenção especial para grandes catástrofes e eventos de grandes magnitudes. Ao estudar o processo pelo qual os atores políticos e sociais enxergam e definem os problemas públicos, buscando o estabelecimento de coalizões, expandindo o conflito e o reconhecimento público dessas questões, não passou despercebido o fato de que, em algumas situações, a emergência e a gravidade com a qual determinados eventos surgem alteram a lógica de funcionamento do processo de atenção e de 
priorização de problemas e de produção de políticas. Grandes catástrofes naturais, como tsunamis e furacões, incêndios e alagamentos de grandes dimensões, ou mesmo eventos inesperados e de grande impacto social, ambiental e econômico, como acidentes nucleares, em minas de carvão ou a queda de aviões, são exemplos de situações que irrompem na agenda governamental com alto grau de prioridades, sem tempo para negociações, uso de símbolos ou de mecanismos de veto.

Tais eventos, chamados de focusing events por John Kingdon (1984), mas que também aparecem sob o termo de triggerin events em Baumgartner e Jones (1993), modificam substancialmente o processo de políticas públicas, do reconhecimento à dinâmica de formulação de políticas públicas. Nesta seção buscaremos identificar a pandemia da Covid-19 como sendo um evento focalizador (BRASIL; CAPELLA, 2020;) apontando gaps, nos casos brasileiro e internacional, que poderão ser investigados em novas agendas de pesquisas.

Os eventos focalizadores, de acordo com Birkland (1997), são raros, imprevisíveis e potencialmente danosos. Geralmente estão vinculados a questões sobre as quais não seria totalmente possível prever o ocorrido a ponto de minimizar ou anular os danos e impactos causados. No entanto, uma distinção precisa ser feita quando trabalhamos com a ideia de eventos focalizadores. Diferentemente de grandes eventos sociais e esportivos, como os Jogos Olímpicos, por exemplo, que têm curta duração e mobilizam diversos setores sociais políticos e econômicos, os eventos focalizadores não são planejados ou esperados. Ainda que a responsabilidade dos representantes não seja nula em eventos como esses, não se pode confundir os problemas e excepcionalidades causados pela falta de controle e de planejamento de grandes eventos, com a ocorrência de eventos repentinos e inesperados como os mencionados. Para Birkland (1997), os eventos focalizados ainda contam com outras características que vão além da sua imprevisibilidade - eventos repentinos, raros e não planejados. Eles são eventos que afetam grande número de pessoas ao mesmo tempo e cujos efeitos prejudiciais se revelam tanto de forma imediata como apresentam danos futuros potencialmente maiores decorrentes da sua ocorrência.

A focusing event is an event that is sudden; relatively uncommon; can be reasonably defined as harmful or revealing the possibility of potentially greater future harms; has harms that are concentrated in a particular geographical area or community of interest; and that is known to policy makers and the public simultaneously. Defining focusing events in this way guides the researcher in selecting the appropriate domains for 
studying focusing events, and helps us to understand more clearly why some events are more intensely "focal" than others". (BIRKLAND, 1997).

Os eventos focalizadores ganham atenção de forma mais repentina e mais rapidamente do que problemas como crimes ou problemas estruturais, como a falta de vagas em creches ou o aumento do percentual de desemprego num país, por exemplo. Os danos óbvios causados por eventos focalizadores destacam a existência de problemas aos quais o governo ou outras instituições precisam responder de forma urgente. Da mesma forma, a narrativa construída para explicar, propor ou mesmo refutar a existência dos problemas anteriormente citados não segue o mesmo padrão nem a mesma velocidade daquela necessária para tratar dos danos imediatos causados por esses eventos focalizadores. Isso não quer dizer que esses problemas sejam maiores do que os demais existentes na sociedade. No entanto, significa dizer que a sua urgência, o seu potencial de atrair e concentrar a atenção de atores, como os formuladores de políticas, a mídia e a opinião pública, os colocam como prioritários no seletivo processo de definição de agenda.

A Pandemia da Covid-19 afetou sociedades de todo o mundo, fazendo com que líderes adotassem diversas medidas de urgência, quer seja para prevenir e reduzir a velocidade de contágio, quer seja para garantir a sobrevivência e a dignidade de seu povo diretamente afetado pelas restrições necessárias. Nesse processo, variadas políticas públicas e instrumentos foram utilizados para superar a crise causada. Guiados por órgãos internacionais, como a OMS (Organização Mundial da Saúde) e seus grupos de trabalhos específicos para a Covid-19, algumas das ações mais imediatas foram utilizadas em sociedades e países diferentes. Sistemas de ensino à distância, home-office, fechamento de fronteiras, alteração de horários de comércio, uso obrigatório de máscaras, distanciamento social são exemplos de recomendações que seguiram de forma padronizada em diversos países e sociedades durante a pandemia. Outras alternativas adotadas, no entanto, mais particulares de cada país e sistemas políticos, podem ser foco de estudos futuros que busquem entender como a pandemia e as mudanças no sistema de reconhecimento de problemas e seleção de alternativas conseguiram inserir difíceis pautas na onda da crise. No caso brasileiro, por exemplo, a renda emergencial surgiu como uma alternativa viável que, muito dificilmente, seria considerada como prioritária em situações de não crise. (). Qual o potencial de inserção de pautas que a urgência na produção de respostas pode gerar? Quais foram os caminhos adotados para a formulação e implementação dessas políticas? 
Para o futuro, perguntas ainda não respondidas devem ser foco de estudos que consideram a permanência e a extinção de políticas formuladas para responder a problemas emergenciais. O sistema de educação à distância funcionou de forma satisfatória a ponto de ser uma alternativa viável para o desenho da educação pública mesmo fora da pandemia? No campo do trabalho, o que a experiência do home-office pode demonstrar sobre a produtividade, os custos e as relações profissionais para o futuro? Quais características e indicadores foram decisivos no processo de continuidade e descontinuidade de políticas adotadas de forma emergencial? Essas perguntas nos levam ao segundo modelo analítico com forte potencial para guiar estudos sobre políticas públicas em tempos de crise, o Aprendizado e Políticas baseadas em Evidências (policy leraning and evidence-based policy making)

\section{Aprendizado e Políticas baseadas em Evidências}

As raízes do policy learning podem ser observadas nos estudos de Heclo (1974) e na profunda difusão e mapeamento dessa lente analítica feitos por Hall (1993) e Dunlop e Radaelli (2013). Nessa abordagem, o destaque reside na aquisição e utilização do conhecimento e de evidências no envolvimento e no aprendizado baseado em valores, crenças e ideias para a análise do processo de produção das políticas públicas. De acordo com Hall (1993, p. 278), o aprendizado "ocorre quando os indivíduos assimilam uma informação nova, incluindo aquela baseada em experiência, e a aplicam em suas ações subsequentes. [...] $\mathrm{O}$ aprendizado é observado quando a política muda como resultado desse processo".

A importância da evidência aumentou substancialmente no processo de tomada de decisão das democracias contemporâneas. Como consequência, muitos estudiosos têm desviado sua atenção para a análise de como os dados científicos podem contribuir para o aprimoramento das políticas públicas. A ideia central por trás do aprendizado inclui a apropriação de novas informações, obtidas ao longo do processo, que podem mudar ou ratificar os sistemas de crenças já existentes. É uma abordagem que valoriza o processo de tomada de decisões bem informadas sobre políticas, programas e projetos, colocando as melhores evidências disponíveis no centro do desenvolvimento e implementação de políticas públicas. Esse aprendizado permite tanto mudanças mais radicais em políticas públicas quanto mudanças discretas, que envolvem ampliação e ajustes. A abordagem do policy learning inclui uma série de estudos dedicados aos processos de difusão e transferência de políticas 
públicas, ao processo de produção de políticas baseado em evidências e, em muitos estudos, aparece vinculado ao modelo de Coalizões de Defesa (ACF Advocacy Coalition Framework) como forma de operação de sistemas de crenças e valores (WEISS; , 2008; SABATIER;, 1999)

É importante retomarmos aqui, sobretudo quando falamos de difusão e transferência em políticas públicas, a forma pela qual entidades internacionais, como a OMS, coordenaram e difundiram recomendações ao longo da pandemia da Covid-19, servindo de base não apenas para a difusão de informação, de dados oficiais e acompanhamento do número de casos e de mortes no mundo, mas também analisando e recomendando ações baseadas em experiências anteriores. $\mathrm{O}$ aprendizado sobre a doença bem como o aprendizado sobre as políticas empregadas pelos países que vivenciaram a primeira onda de contágios e mortes, como China, Espanha e Itália, produziram conhecimento e evidências sobre as ações tomadas, apontando e separando o que funcionou e o que não surtiu efeito no controle do contágio e de suas consequências. Em muitos países, como Finlândia e Nova Zelândia, por exemplo, a antecipação na produção de políticas restritivas, de isolamento, fechamento de fronteiras e informações sobre uso de máscaras, higienização de mãos, por exemplo, fez com que o impacto da pandemia, nesses países, fosse drasticamente inferior quando comparado à situação daqueles que vivenciaram a doença na primeira onda, ou daqueles países cujos governantes optaram por não acatar as recomendações provenientes do aprendizado e das evidências anteriores, como os casos do Brasil e dos Estados Unidos.

De acordo com especificações elaboradas por Dunlop e Radaelli (2013), o caso poderia ser analisado pelas lentes do chamado aprendizado epistêmico, caracterizado pela centralização da autoridade em experts na área, formalizada por decisões baseadas em evidências em que há recomendações diretas com o objetivo de reduzir o escopo do conflito e alcançar soluções para o problema. $\mathrm{O}$ aprendizado obtido por meio da experiência anterior, dos resultados obtidos na formulação de políticas em contextos próximos seria capaz de sustentar um sistema baseado na relação entre as evidências e os resultados desejados na busca por soluções aos problemas decorrentes da crise.

Esse debate se faz necessário e é aqui apontado como indicativo de agendas futuras em pesquisas de políticas públicas sobre a Covid-19, uma vez que evidencia, mesmo em situações de risco iminente, a existência de espaço para o contraditório e para a contestação de ideias e valores. As lentes centradas no papel das ideias e nas suas relações com as instituições e atores mostram, nesse e em outros tantos casos já estudados pela literatura especializada, o quão forte 
e importante é a noção de sistemas de crenças e construção social de problemas e alternativas para a formulação de políticas públicas. A formulação de políticas baseadas em evidências assim como a difusão ou mudança de políticas públicas baseadas em aprendizado não isolam a pressão feita por outros grupos de pressão com ideias e valores distintos. Mesmo durante a pandemia, as estratégias de negação, de desresponsabilização e de refutação da ciência e das evidências foram imensamente utilizadas como forma de formação de coalizões e de justificativas para a não ação do Estado. O mesmo processo é percebido quando observamos as reações e o posicionamento de grupos sobre as políticas de vacinação, que deve gerar, num futuro breve, excelentes e necessários estudos de caso sobre sistemas de crenças e aprendizado em políticas, quando observamos o fenômeno da pandemia Covid-19.

\section{Conclusão}

Este artigo procurou demonstrar brevemente algumas linhas teóricas que podem servir de base analítica para novos estudos que buscam melhor compreender o fenômeno da pandemia da Covid-19 nos processos de políticas públicas. Longe de restringir o campo analítico aos temas abordados, a agenda que se constrói em torno do tema é ampla e, ao mesmo tempo, difícil de mapear, dada a intersetorialidade do campo e da magnitude do evento e suas consequências. Estudos sobre as capacidades estatais e as formas de implementação de políticas públicas são exemplos de um crescente campo do conhecimento não considerado nessa proposta. Pesquisas empíricas e teóricas nos campos setoriais da educação, saúde, trabalho, assistência social e afins também deverão dominar os estudos sobre a relação entre políticas públicas e a pandemia nos anos que se seguem.

Aqui, no entanto, destacamos duas vertentes ainda pouco conhecidas ou trabalhadas pela literatura nacional. A primeira delas é a que trata dos eventos focalizadores. Presente desde as primeiras concepções teóricas sobre os estudos de agenda-setting, os focusing events parecem ter pouca aplicação no caso brasileiro, sobretudo quando consideramos o seu histórico analítico sobe eventos naturais, como tsunamis, furacões e terremotos, eventos com maior ocorrência nos Estados Unidos, de onde a teoria é originária. A pandemia, entendida aqui como um evento repentino, imprevisível, global e de forte impacto, tem se mostrado uma variável relevante nos estudos da formação da agenda e da formulação de políticas no Brasil, na medida que consegue inserir 
com maior rapidez pautas caras e que dificilmente seriam consideradas fora do ambiente de crise. Entender a forma como os atores se posicionam e se movimentam, as imagens construídas e as capacidades lançadas para possibilitar a produção de políticas emergenciais deve se tornar foco de estudos futuros, assim como a permanência ou suspensão de políticas implementadas para além da crise.

Por outro lado, a literatura de policy learning e evidence-based policy making agrega outros componentes aos estudos de políticas públicas relacionados à pandemia. Qual o papel das evidências e do aprendizado no processo de políticas públicas? O que as experiências vividas em outros países e sistemas pode gerar de informação e de direcionamento para lidar com problemas novos, mas catastróficos? Observamos brevemente, em alguns casos específicos, que a aprendizagem e as evidências podem ou não ser utilizadas como fonte para a produção de políticas. A negação, as ideologias e as ferramentas de bloqueio existem e podem ser usadas à revelia dos benefícios aprendidos a partir de evidências e vivências anteriores. $\mathrm{O}$ impacto causado em diferentes nações e a forma de atuação de diferentes líderes pode ser capaz de demonstrar, em agendas de pesquisas futuras, a importância do aprendizado e do uso de evidências em todo o processo de políticas públicas.

\section{Referências}

BAUMGARTNER, Frank; JONES, Bryan.

(1993). Agendas and instability in American politics. Chicago: University of Chicago Press.

BIRKLAND, Thomas.

(1997). After Disaster. agenda-setting, public policy and focusing events. Washington D.C., Georgetown University Press.

BIRKLAND, Thomas.

(2006). Lessons of Disaster. Georgetown University Press.

BIRKLAND, Thomas.

(2005). An Introduction to the Policy Process: Theories, Concepts, and Models of Public Policy Making M.E. Sharpe.

BRASIL, F. G.; CAPELLA, Ana C. N.

(2020). Janelas escancaradas: o potencial da Pandemia na mudança em políticas públicas. Boletim: Cientistas sociais e o coronavirus. ISSN
2675-4339 Disponível em: http://www.anpocs.com/index.php/cienciassociais/destaques/2325-boletim-semanal. Acesso em: 3 jul. 2020

CAPELLA, Ana C. N.

(2007). Perspectivas Teóricas sobre o Processo de Formulação de Políticas Públicas". In Hochman, G.; Arretche, M.; Marques, E. Políticas Públicas no Brasil. Rio de Janeiro, Fiocruz.

CAPELLA, Ana Cláudia; BRASIL, Felipe Gonçalves. (2016). Análise de políticas públicas: uma revisão da literatura sobre o papel dos subsistemas, comunidades e redes. Novos estudos - CEBRAP. n.101 p.57-76.

COBB, R. W.; ELDER, C. D.

(1972). Participation in American Politics: The dynamics of agenda building. Boston: Allyn and Bancon. 
(1983). Participation in American Politics: the Dynamics of Agenda Building. $2^{\text {nd }}$ Ed. Baltimore: The Johns Hopkings Press.

COBB, R. W.; ELDER, C. D; ROSS, J. K.; ROSS, M. H. (1976 mar.). Agenda Building as a comparative political process. American Political Science Review, v. 70, n. 1, p. 126-138.

COHEN, B. C.

(1963). The press and foreign policy. Princeton, $\mathrm{NJ}$ : Princeton University Press.

COHEN, M. D.; MARCH, J. G.; OLSEN, J. P.

(1972 mar.). A Garbage Can Model of Organizational Choice. Administrative Science Quarterly, Ithaca, v. 17, n. 1, p. 1-25.

DUNLOP, C. A.; RADAELLI, C. M.

(2013). Systematising Policy Learning: From Monolith to Dimensions. Political Studies, vol. 61, n.3, p. 599-619.

(2018). The lessons of policy learning: Types, triggers, hindrances and pathologies. Policy and Politics, vol. 46, n. 2, p. 255-272

HALL, P. A.

(1993). Policy Paradigms, Social Learning, and the State: The Case of Economic Policymaking in Britain. Comparative Politics, vol. 25, n. 3, p. 275-296.

HECLO, Hugh.

(1974). Modern Social Politics in Britain and Sweden: From Relief to Income Main-tenance. New Haven: Yale University Press.

KINGDON, J.

(1984). Agendas, Alternatives, and Public Policies. New York: Harper Collins.

(2003). Agendas, Alternatives, and Public Policies. $3^{\text {st }}$. Ed. New York: Harper Collins.

KLAPPER, J. T.

(1960). The Efects of Mass Communication. New York: Free Press.

LAZARSFELD, P. F.; BERELSON, B.; GAUDET, H. (1944). The People's Choice: How the Voter Makes Up His Mind in a Presidential Campaign. $2^{\text {nd }} \mathrm{Ed}$. New York: Columbia University.
LIPPMANN, W.

(1922). Public opinion. New York: Harcourt Brace.

MCCOMBS, M.; SHAW, D.

(1972). The agenda-setting function of mass media. Public Opinion Quarterly, 36, p. 176195.

MCCOMBS, M.E.; SHAW, D.L.

(1993). The evolution of agenda-setting research: Twenty-five years in the marketplace of ideas. Journal of Communication, vol. 43, n. 2, p. 58-67.

SABATIER, P.A.

(1999). Theories of the Policy Process. Boulder: Westview Press, p.117-166.

TRAQUINA, Nelson.

(1995). 0 paradigma do agenda-setting:

Redescoberta do poder do jornalismo". In:

Revista Comunicação e Linguagens. Lisboa:

Cosmos, n.21-2.

WEIBLE; P. A.; SABATIER (Eds.).

(2014) Theories of the Policy Process. 4th ed., p. 135-171. New York, NY: Routledge.

WEIBLE, C. M.

(2008). Expert-based information and policy subsystems: A review and synthesis. Policy Studies Journal, vol. 36, n. 4, p. 615-635.

(1998). Have We Learned Anything New About the Use of Evaluation? American Journal of Evaluation, vol. 19, n. 1, p. 21-33

\section{Recebido em}

dezembro de 2020

\section{Aprovado em}

dezembro de 2020 